\title{
Thymocytes maintain immune activity through telomere elongation in rats under hypoxic conditions
}

\author{
YAPING WANG ${ }^{1,2^{*}}$, ZHEN ZHAO $^{1 *}$, YINGZHONG YANG $^{2}$, YANXIA ZHAO $^{2}$ and RI-LI GE ${ }^{2}$ \\ ${ }^{1}$ Department of Digestion, Qinghai Provincial People's Hospital, Xining, Qinghai 810007; \\ ${ }^{2}$ Research Center for High Altitude Medicine, Medical College of Qinghai University, \\ Xining, Qinghai 810001, P.R. China
}

Received October 3, 2014; Accepted September 1, 2015

DOI: $10.3892 / \mathrm{etm} .2015 .2754$

\begin{abstract}
The main purpose of the present study was to investigate the change in thymocyte telomere length of rats exposed to different hypoxic conditions for different periods of time, as well as its effect on the immune system. A total of 110 male Wistar rats were randomly assigned to one of the three following groups: i) Sea level (SL) group, in which 10 rats were maintained at an altitude of $10 \mathrm{~m}$; ii) moderate altitude (MA) group, in which 50 rats were maintained at an altitude of 2,260 $\mathrm{m}$ and then randomly sacrificed on days $1,3,7,15$ and 30 ( $n=10$ each); and iii) simulated high altitude (SHA) group, in which 50 rats were maintained at a simulated altitude of $5,000 \mathrm{~m}$, and then randomly sacrificed on days $1,3,7,15$ and 30 ( $n=10$ each). The morphological changes of the thymus were observed, while the telomere length, the mRNA and protein expression levels of telomerase reverse transcriptase (TERT), and the peripheral blood lymphocyte count were measured. The results indicated that hypoxia induced morphological changes and apoptosis in thymocytes, as well as atrophy of the thymus tissue, and resulted in a significant increase in telomere length and TERT mRNA and protein expression levels. This effect appeared to be more pronounced in the SHA group compared with that in the MA group; however, no statistically significant changes were observed in the peripheral blood lymphocyte count. Based on these findings, the hypoxia-associated loss of thymic function appears to be only quantitative and not qualitative, and the thymus may be able to maintain its immune function even under hypoxic conditions.
\end{abstract}

Correspondence to: Dr Ri-Li Ge, Research Center for High Altitude Medicine, Medical College of Qinghai University, 16 Kunlun Road, Xining, Qinghai 810001, P.R. China

E-mail: geriligao@hotmail.com

*Contributed equally

Key words: telomere, hypoxia, telomerase reverse transcriptase, thymocytes

\section{Introduction}

Telomeres are protective structures at the ends of eukaryotic chromosomes, which consist of tandem arrays of hexameric repeats (TTAGGG) bound to specific proteins. Telomeres play a critical role in the maintenance of the structural integrity of the genome, as they can prevent chromosomes from nucleolytic decay, fusion and atypical recombination (1). In human somatic cells, telomeres shorten by 30-200 bp with each mitotic cell division (2). Thus, telomere length is expected to be an effective indicator of the mitotic history of a cell, since it reflects the cumulative effect of cell divisions occurring in vitro upon stimulation and in vivo due to aging $(3,4)$. Telomere length is also a determining factor for the residual life span of normal somatic cells, since further cell division is not possible in these cells when telomeres shorten to a critical length $(5,6)$. For this reason, telomere length has long been proposed as a biomarker of aging (7).

The functional activity of the immune system depends on the degree of lymphocyte proliferation and clonal expansion during development, activation and differentiation (8). Telomere maintenance is of paramount importance for dynamic cellular systems, such as the immune system. The thymus is a primary lymphoid organ responsible for T-lymphocyte differentiation and maturation, which provides an inductive microenvironment in which bone marrow-derived progenitors undergo proliferation. Thymocytes differentiate into mature $\mathrm{T}$ cells that are essential for the immune response against infection (9-11). It has also been suggested that intrathymic maturation and selection of $\mathrm{T}$ cells are associated with telomerase activity, which allows $\mathrm{T}$ cells to acquire telomere sequences that are long enough to undergo several rounds of replication (12).

A variety of environmental and cellular factors have been shown to affect the rate of telomere attrition and telomerase activity, as well as the number of cell divisions (history of division), such as low temperature, dry, strong ultraviolet radiation, smoking, diet and psychological stress (13-16). In recent years, the effect of hypoxia on telomere length has become a topic of increasing interest and debate. Previous studies have indicated that the telomere length varies considerably among hypoxia levels and cell types (17-19), and thus telomere length in lymphocytes may indicate the extent to which hypoxia affects the immune system. 
Our previous study detected telomere length of leukocytes in rats exposed to different hypoxic altitudes. The results showed that the leukocyte telomeres were significantly elongated in a mildly hypoxic environment, as opposed to a highly hypoxic environment. To the best of our knowledge, there are no previous studies investigating the impact of different hypoxic altitudes on thymocyte telomere length and telomerase reverse transcriptase (TERT) expression.

In the present study, the telomere length and telomerase reverse transcriptase (TERT) of rat thymocytes exposed to different altitudes $(10,2,260$ and 5,000 m) was measured after different periods of time (1, 3, 7, 15 and 30 days), and their effect on the rat immune system was investigated, with a focus on the dynamic behavior of telomere length and TERT in thymocytes.

\section{Materials and methods}

Animal experiments. All animal experiments were approved by the Institutional Animal Ethics Committee of the Medical College of Qinghai University (Xining, China). A total of 110 male Wistar rats of the same age (6 weeks) and weight (50-180 g) were used in the present study. The rats were maintained in a controlled environment at a temperature of $18-20^{\circ} \mathrm{C}$ on a 12-h light/dark cycle with unlimited access to food and water, and were randomly assigned to one of the following three groups: i) Sea level (SL) group, in which 10 rats were maintained at an altitude of $10 \mathrm{~m}$ in Nanjing, China, and immediately sacrificed on day 1 ; ii) moderate altitude (MA) group, in which 50 rats were maintained at an altitude of 2,260 $\mathrm{m}$ in Xining, China, and then randomly sacrificed on days 1, 3, 7, 15 or 30 ( $\mathrm{n}=10$ each); and iii) simulated high altitude (SHA) group, in which the remaining 50 rats were maintained in an 8x3x3-m hypobaric chamber (DYC-3000; Guizhou Fenglei Aviation Armament Co., Ltd., Anshun, China) with a simulated altitude of 5,000 $\mathrm{m}$ in Xining, China, and were then randomly sacrificed on days $1,3,7,15$ or 30 ( $n=10$ each). The rats were sacrificed under Nembutal anesthesia $(40 \mathrm{mg} / \mathrm{kg}$, intraperitoneally; H. Lundbeck A/S, Copenhagen, Denmark), and their thymus glands were excised and weighed. Thymus specimens $\left(0.3 \mathrm{~cm}^{3}\right)$ were fixed for histopathological analysis as described in a subsequent section, and the remainder of the thymus was fresh-frozen and stored in liquid nitrogen at $-80^{\circ} \mathrm{C}$ for molecular analysis.

Peripheral blood lymphocyte count and percentage. The peripheral blood lymphocyte count and percentage were measured using an automatic blood cell analyzer (BC-2300; Mindray Medical International Ltd., Wuhan, China) following the manufacturer's instructions.

Thymus index and histopathological analysis. The rats were anesthetized using Nembutal $(40 \mathrm{mg} / \mathrm{kg}$, intraperitoneally; H. Lundbeck A/S), and their thymuses were excised and weighed. The thymus index was expressed as the thymus weight relative to the body weight. A $0.3-\mathrm{cm}^{3}$ sample of the thymus was fixed in $4 \%$ paraformaldehyde, routinely embedded in paraffin, sectioned, and stained with hematoxylin and eosin (Nanjing Biological Co., Jiangsu, China) for histopathological analysis.
Measurement of telomere length using quantitative polymerase chain reaction $(q P C R)$. The thymus tissues were ground and passed through a 200-mesh sieve, centrifuged at $400 \mathrm{x}$ g for $10 \mathrm{~min}$ at $4^{\circ} \mathrm{C}$, washed twice with phosphate-buffered saline and counted using a microscope (Olympus Corporation, Tokyo, Japan). The genomic DNAs were isolated from $10^{6}-10^{7}$ cells using a DNA extraction kit (Tiangen Biotech Co., Ltd., Beijing, China) following the manufacturer's instructions, and dissolved in Tris-EDTA buffer ( $\mathrm{pH}$ 8.0). The concentration was measured using a DU 800 spectrophotometer (Beckman Coulter, Inc., Fullerton, CA, USA). The ratio of OD260 to OD280 was 1.8-2.0 and the DNA concentration was $50 \mathrm{ng} / \mu \mathrm{l}$.

qPCR reactions were performed using $\mathrm{SYBR}^{\circledR}$ Select Master Mix with an ABI 7500 system (Applied Biosystems, Carlsbad, CA, USA) in a total volume of $20 \mu \mathrm{l}$ containing $2 \mu \mathrm{l}$ DNA template. The PCR plate was incubated at $95^{\circ} \mathrm{C}$ for $30 \mathrm{sec}$, followed by 40 cycles of $95^{\circ} \mathrm{C}$ for $5 \mathrm{sec}$ and $60^{\circ} \mathrm{C}$ for $34 \mathrm{sec}$. All samples were tested in triplicate, and the amplification efficiency was compared with that of the angiotensin type 1 (AT1) receptor reference gene and was found to be similar. The primers used for the amplification of telomeres were as follows: Tel-1, forward (270 nmol) 5'GGTTTTTGAGGGTGAGGG TGAGGGTGAGGGTGAGGGT-3' and reverse (900 nmol) 5'-TCCCGACTATCCCTATCCCTATCCCTATCCCTATCC CTA-3'; AT1, forward (400 nmol) 5'-ACGTGTTCTCAGCAT CGACCGCTACC-3'; and reverse (400 nmol) 5'-AGAATG ATAAGGAAAGGGAACAAGAAGCCC-3'. Primers were synthesized by Invitrogen (Grand Island, NY, USA).

The relative telomere length was expressed as the T/S ratio, where $\mathrm{T}$ is the amount of telomere and $\mathrm{S}$ the amount of a single gene copy of the AT1 receptor. The $2^{-\Delta \Delta \mathrm{Ct}}$ method was used to calculate the $\mathrm{T} / \mathrm{S}$ ratio.

Protein and $m R N A$ expression levels of TERT. Total RNA was extracted from the thymocytes and purified using TRIzol ${ }^{\circledR}$ reagent (Invitrogen Life Technologies) following the manufacturer's instructions. The RNA concentration was measured using the DU 800 spectrophotometer. cDNA synthesis was performed with $2 \mu \mathrm{g}$ total RNA, and this cDNA was used as a template in qPCR. PCR reactions were performed using SYBR $^{\circledR}$ Select Master Mix with an ABI 7500 system in a total volume of $20 \mu \mathrm{l}$. The PCR plate was incubated at $50^{\circ} \mathrm{C}$ for $2 \mathrm{~min}$ and $95^{\circ} \mathrm{C}$ for $2 \mathrm{~min}$, followed by 40 cycles of $95^{\circ} \mathrm{C}$ for $15 \mathrm{sec}$ and $60^{\circ} \mathrm{C}$ for $1 \mathrm{~min}$. The amplification efficiency of TERT was compared with that of the $18 \mathrm{~S}$ rRNA reference gene and was found to be similar. The primers used for the amplification of TERT were as follows: TERT forward (400 nmol), 5'-GACATGGAGAACAAGCTGTTTGC-3', and reverse (400 nmol), 5'-ACAGGGAAGTTCACCACTGTC-3'; 18S rRNA forward (400 nmol), 5'-AGTGATCCCCGAGAA GTTT-3', and reverse (400 nmol), 5'-GCTTTCCTCAACACC ACAT-3' (Invitrogen). The $2^{-\Delta \Delta C q}$ method was used to calculate the relative expression levels.

Total protein of rat thymocyte cell suspension was extracted and measured by the BCA Protein Assay kit (Pierce Biotechnology, Inc., Rockford, IL, USA) and the protein levels of TERT were detected using an ELISA kit (Cloud-Clone Corp., Houston, TX, USA) following the manufacturer's instructions. 

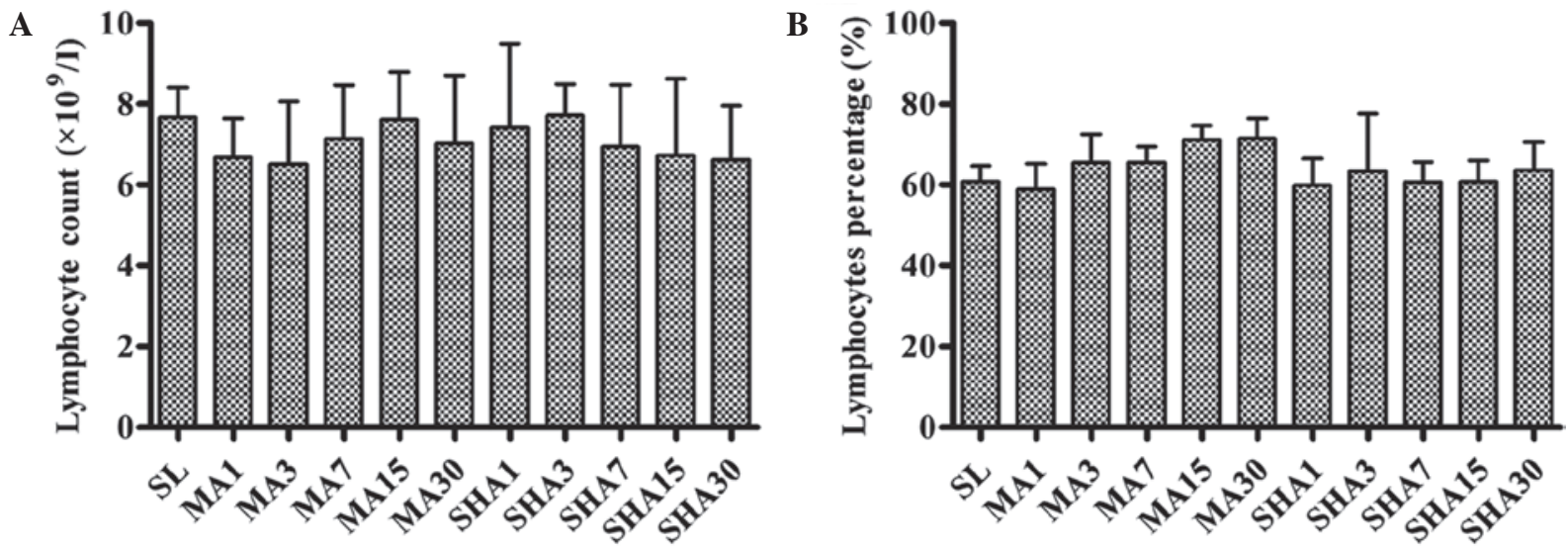

Figure 1. (A) Peripheral blood lymphocyte count and (B) lymphocyte percentage in various groups. Data are presented as the mean \pm standard deviation (n=10 each). SL, sea level; MA, moderate altitude; SHA, simulated high altitude.

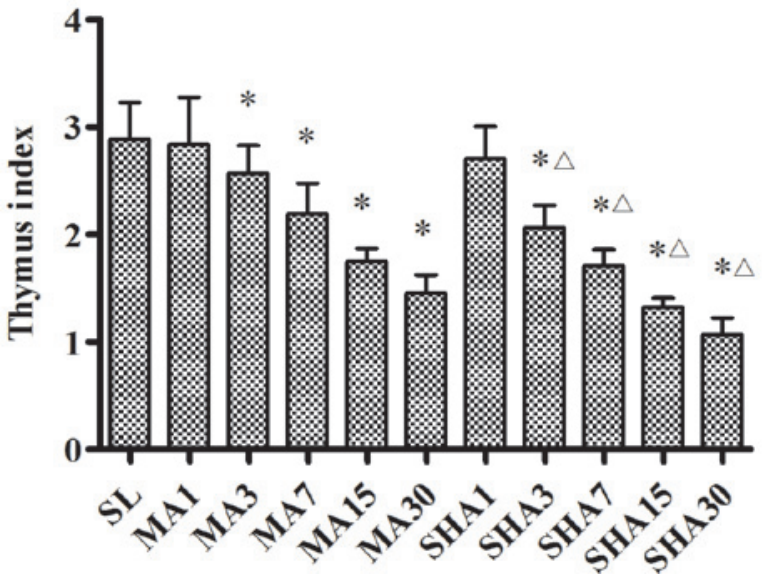

Figure 2. Thymus index of various groups. Data are presented as the mean \pm standard deviation $\left(\mathrm{n}=10\right.$ each). ${ }^{*} \mathrm{P}<0.05$ vs. SL group; ${ }^{\Delta} \mathrm{P}<0.05$ vs MA group of same exposure. SL, sea level; MA, moderate altitude; SHA, simulated high altitude.

Statistical analysis. Data were analyzed using SPSS software, version 17.0 (SPSS, Inc., Chicago, IL, USA). All data are presented as the mean \pm standard deviation. Statistical analysis was performed using one-way analysis of variance followed by a least significant difference post-hoc test. $\mathrm{P}<0.05$ was considered to indicate a statistically significant difference.

\section{Results}

Hematological changes. As shown in Fig. 1, no statistically significant changes were observed in the peripheral blood lymphocyte count and percentage in rats exposed to different altitudes for different periods of time $(\mathrm{P}>0.05)$. The thymus is the primary lymphoid organ of cellular immunity responsible for T-lymphocyte choice and output. No changes means that the body cellular immunity has not been obviously damaged, the rat thymus still plays the effective immune function under hypoxic conditions.

Morphological changes of thymus. It is known that the degree of hypoxia is associated with altitude, higher altitude results in increased levels of hypoxia. According to the international standard, the moderate altitude used in the preset study $(2,260 \mathrm{~m})$ is associated with mild hypoxia, while simulated high altitude $(5,000 \mathrm{~m})$ is associated with severe hypoxia. Fig. 2 shows that the thymus index of rats exposed to hypoxic conditions decreased with increasing exposure time $(\mathrm{P}<0.05)$. This decrease in the thymus index appeared to be more pronounced in the SHA group compared with that in the MA group $(\mathrm{P}<0.05)$. The decreased thymus index indicated that the thymocyte number reduction and thymus tissue atrophy occurred as a result of the hypoxic conditions.

As shown in Fig. 3A, in the SL group, the cortex was thick and distinct corticomedullary demarcation was observed. The lymphocytes were small and densely clustered in the cortex, with deeply stained nuclei. Thymocyte apoptosis or epithelial cell hyperplasia were not observed, and there was no abnormal distribution or hyperemia of mesenchymal vessels; however, thymic corpuscles were observed. In the MA group, the medulla expanded, and the cortex became thin with distinct corticomedullary demarcation. The thymocytes were small and densely clustered in the cortex, with deeply stained nuclei. Thymocyte apoptosis was rarely observed in the MA group. Hyperplastic and hypertrophic epithelial cells were present in the cortex and medulla, while thymic corpuscles were also identified. Furthermore, the mesenchymal vessels were found to be expanded and hyperemic, with small patches of hemorrhage. The parenchyma of thymic lobules was found to be atrophied and infiltrated by adipose tissue. The mild tissue damage and thymocyte apoptosis observed as a result of moderate altitude was not evidently exacerbated following an extension of hypoxic exposure time, and there were no significant differences between the five times.

In the SHA group, the medulla expanded, the cortex became thin with distinct corticomedullary demarcation, and the thymocytes were large with lightly stained nuclei. The chromatin was thick, and significant thymocyte apoptosis was observed. Focal loss of thymocytes was identified, and loose plaque was found in the subcapsule and cortex. Furthermore, hyperplastic and hypertrophic epithelial cells were present in the cortex and medulla and thymic corpuscles were also observed. Mesenchymal vessels were found to be expanded and hyperemic, with small patches of hemorrhage. Certain thymus tissues were found to be atrophied and to have been 

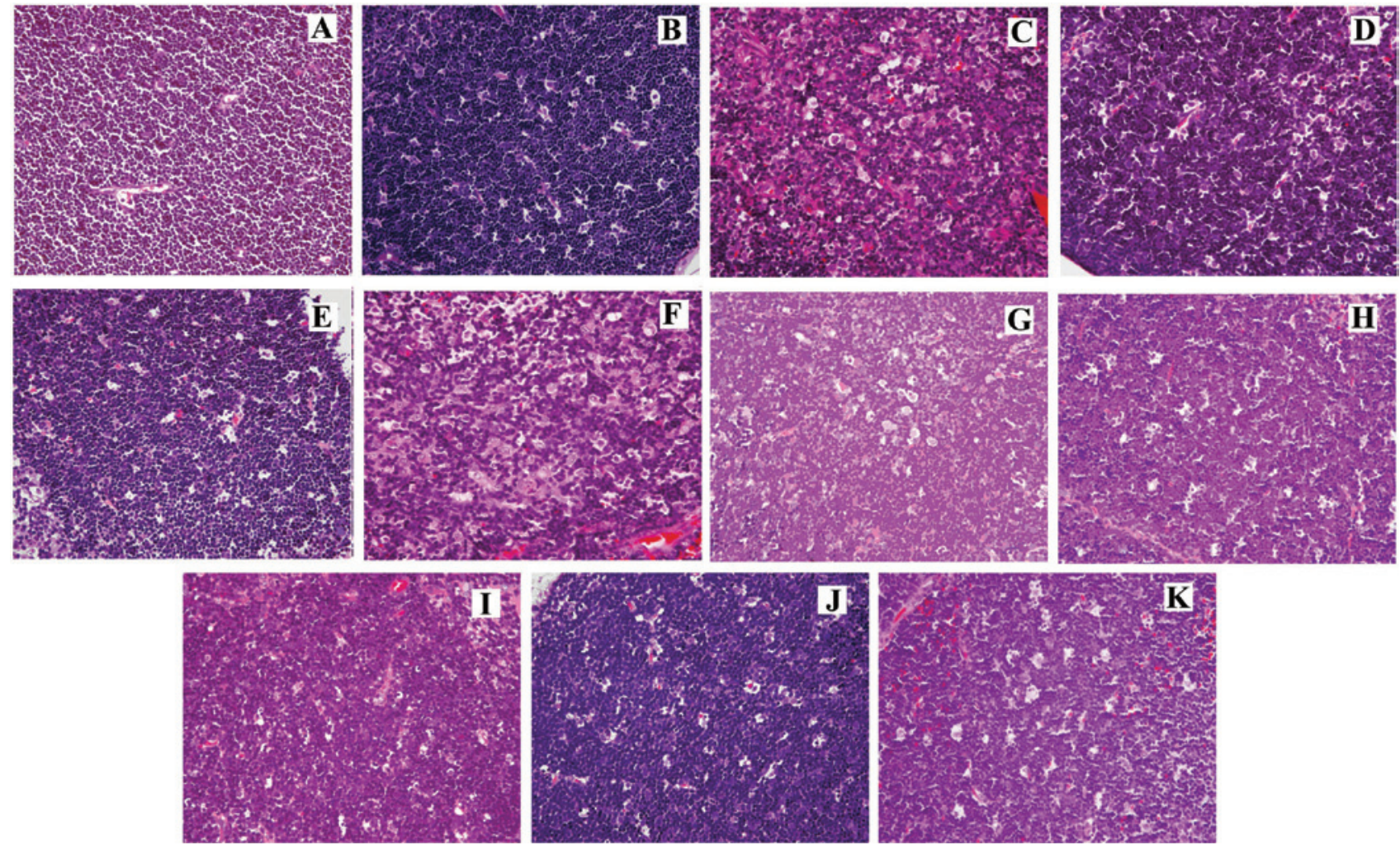

Figure 3. Histological changes (hematoxylin and eosin stain; magnification, x400). (A) In the SL group, the lymphocytes were small and densely clustered in the cortex with deeply stained nuclei. No thymocyte apoptosis was observed. In the MA group on days (B) 1, (C) 3, (D) 7, (E) 15 and (F) 30 , the cortex became thin, and the thymocytes were small and densely clustered in the cortex. Thymocyte apoptosis was rarely observed. The parenchyma of thymic lobules was atrophied and infiltrated by adipose tissue. In the SHA group on days (G) 1 (H) 3 (I) 7 (J) 15 (K) 30, the cortex was thin, and the thymocytes in the cortex were large. Significant thymocyte apoptosis and focal loss of thymocytes were observed, and loose plaque formed in the sub-capsule and cortex. Certain thymus tissues had atrophied and were replaced by adipose tissue.

A

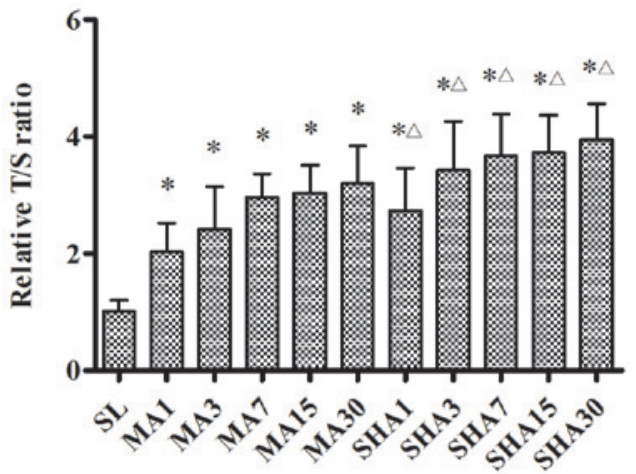

B

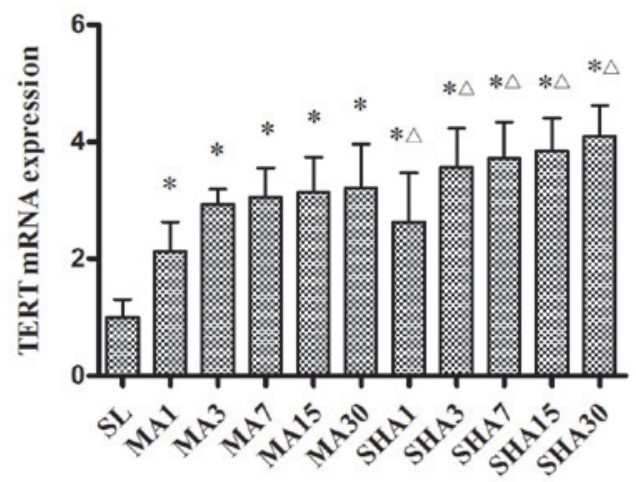

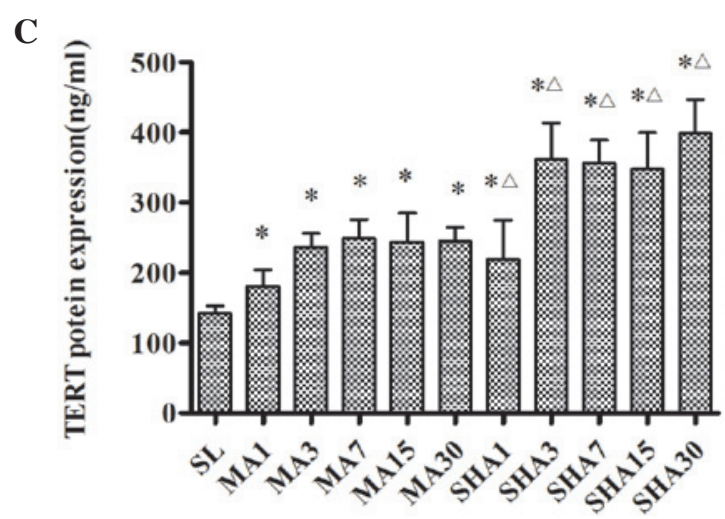

Figure 4. (A) Telomere length, (B) TERT mRNA and (C) TERT protein expression. Telomere length is presented as the relative T/S ratio. The remaining data are presented as the mean \pm standard deviation $(\mathrm{n}=10)$. ${ }^{*} \mathrm{P}<0.05$ vs. SL group; ${ }^{\Delta} \mathrm{P}<0.05$ vs. MA group of same exposure time. TERT, telomerase reverse transcriptase; $\mathrm{T} / \mathrm{S}$, amount of telomere/amount of a single gene copy of AT1 receptor. SL, sea level; MA, moderate altitude; simulated high altitude. 
replaced by adipose tissue. As a result of the extension of hypoxic exposure time, the apoptosis and focal loss of thymocyte under simulated high altitude was gradually exacerbated.

In conclusion, hypoxia induced significant morphological changes and apoptosis in thymocytes, as well as thymus tissue atrophy, and this effect was more apparent in highly hypoxic conditions rather than in mild hypoxic conditions

Telomere length. The relative $\mathrm{T} / \mathrm{S}$ ratios for each group are shown in Fig. 4A. It was evident that telomere length was significantly increased during the first 7 days of exposure to moderate or simulated high altitude $(\mathrm{P}<0.05)$, but with a less significant increase over the next few weeks. Again, this effect tended to be more pronounced in the SHA group than in the MA group $(\mathrm{P}<0.05)$. The results suggested that thymocyte telomere length was elongated gradually in response to an acute hypoxic exposure period, and elongated no further as a result of the chronic hypoxic exposure period.

TERT mRNA and protein expression levels. Fig. 4B and C shows that TERT mRNA and protein expression changed in a manner similar to that observed for the telomere length. TERT mRNA and protein expression were significantly increased in response to hypoxic exposure during the first week $(\mathrm{P}<0.05)$, but remained stable over the next few weeks. It was also noted that this increase was more pronounced in the SHA group than it was in the MA group $(\mathrm{P}<0.05)$. Telomere length was closely associated with TERT expression levels, and was correlated with TERT expression under hypoxic conditions.

\section{Discussion}

In the present study, hypoxia was found to induce morphological changes and apoptosis in thymocytes, as well as atrophy of the thymus tissue, and this effect became more pronounced with the increase in hypoxia. In addition, telomere length was significantly increased in response to hypoxic exposure, while TERT mRNA and protein expression also changed in a similar manner.

Telomerase is a ribonucleoprotein that maintains telomere length in germline and stem cells by adding hexameric TTAGGG repeats to the telomeres, thereby compensating for the progressive loss of telomeric sequences with each cell division $(20,21)$. The protein catalytic subunit of telomerase, known as TERT, adds telomere repeats to the ends of chromosomes through the reverse transcription of an RNA template into DNA (22), and is thus responsible for the maintenance of telomere DNA length, chromosomal stability and cellular immortality (23). It has been shown that the introduction of TERT gene into somatic diploid cells resulted in a significant increase in telomerase activity and telomere length $(24,25)$, which suggests that it has a protective function against genome instability-promoting events (26).

The thymus is the primary lymphoid organ responsible for T-lymphocyte differentiation and maturation. The thymus gland is composed of structural components, including peripheral blood, vascular bed, nervous fibers and connective tissues. During their differentiation, thymocytes interact with antigen presenting cells, such as macrophages and dendritic cells, as well as epithelial cells localized in specific thymic areas.
Intrathymic selection and maturation of $\mathrm{T}$ cells is known to be associated with telomerase activity, allowing thymocytes to acquire telomere sequences that are long enough to undergo several rounds of replication (12). It has been recently demonstrated that the decrease in lymphocyte function accelerated telomere shortening in individuals with prolonged psychological stress $(27,28)$. The shortening of a telomere is accompanied by a host of phenotypic and gene expression changes that result in altered functions (29). Thus, thymocyte function depends, to a great extent, on the telomere length and TERT expression. The telomere length of newly generated $\mathrm{T}$ cells is determined by the telomere length of hematopoietic stem cells and the efficacy of telomere elongation in thymocytes (30).

In the present study, hypoxia induced morphological changes and apoptosis in thymocytes. Notably, no significant changes were observed in the total number of peripheral blood lymphocytes; however, the telomere length, as well as the TERT mRNA and protein expression levels, increased with the increase in altitude. These results suggest that the thymus plays an active role in the selection and development of T cells under hypoxic conditions. Previous studies have investigated the possible molecular mechanisms underlying hypoxia-induced TERT expression, and have indicated that TERT expression is upregulated following the induction of HIF-1 $\alpha$ during exposure to hypoxia (31). Furthermore, HIF-1 $\alpha$ functioned as a key transactivator for the induction of TERT transcription, thereby resulting in elongation of telomeres (32). During exposure to hypoxia, the thymus function per thymocyte unit may remain constant; however, a decrease is observed in the total number of thymocyte units. The hypoxia-associated loss of thymic function, therefore, appears to only be quantitative and not qualitative.

In conclusion, hypoxia was found to induced thymocyte apoptosis and thymus tissue atrophy in rats, resulting in a significant increase in telomere length, as well as in the TERT mRNA and protein expression levels. It was also noted that this effect became more apparent with the increase in hypoxia. All these findings suggest that the hypoxia-associated loss of thymic function appears to only be quantitative and not qualitative. In addition, the thymus may be able to maintain its immune function even under hypoxic conditions. Further studies are required to determine the precise underlying mechanism.

\section{Acknowledgements}

This study was supported by the National Basic Research Program of China (grant no. 2012CB518200), the Program of International Science and Technology Cooperation of China (grant no. 2011DFA32720) and the National Natural Science Foundation of China (grant no. 31160219).

\section{References}

1. Stewart SA and Weinberg RA: Telomeres: Cancer to human aging. Annu Rev Cell Dev Biol 22: 531-557, 2006.

2. Shay JW and Wright WE: Telomerase activity in human cancer. Curr Opin Oncol 8: 66-71, 1996.

3. Lu W, Zhang Y, Liu D, Songyang Z and Wan $M$ : Telomeres - structure, function, and regulation. Exp Cell Res 319: 133-141, 2013.

4. Artandi SE: Telomeres, telomerase, and human disease. N Engl J Med 355: 1195-1197, 2006. 
5. Johnson TE: Recent results: Biomarkers of aging. Exp Gerontol 41: 1243-1246, 2006.

6. Cherkas LF, Aviv A, Valdes AM, Hunkin JL, Gardner JP Surdulescu GL, Kimura M and Spector TD: The effects of socia status on biological aging as measured by white-blood-cell telomere length. Aging Cell 5: 361-365, 2006.

7. Hunt SC, Chen W, Gardner JP, Kimura M, Srinivasan SR, Eckfeldt JH, Berenson GS and Aviv A: Leukocyte telomeres are longer in African Americans than in whites: The national heart, lung and blood institute family heart study and the bogalusa heart study. Aging Cell 7: 451-458, 2008

8. Zhao Wushu. Immune balance study and its clinical significance. Beijing: Science Press. 2005:12-18.

9. Anderson G and Jenkinson EJ: Lymphostromal interactions in thymic development and function. Nat Rev Immunol 1: 31-40, 2001

10. Madhok AB, Chandrasekran A, Parnell V, Gandhi M, Chowdhury D and Pahwa S: Levels of recent thymic emigrant cells decrease in children undergoing partial thymectomy during cardiac surgery. Clin Diagn Lab Immunol 12: 563-565, 2005.

11. Moore SE, Collinson AC, Tamba N'Gom P, Aspinall R and Prentice AM: Early immunological development and mortality from infectious disease in later life. Proc Nutr Soc 65: 311-318, 2006.

12. Brousset P, al Saati T, Zenou RC and Delsol G: Telomerase activity might persist in the human thymus throughout life. Mol Pathol 51: 170-173, 1998.

13. Lung FW, Chen NC and Shu BC: Genetic pathway of major depressive disorder in shortening telomeric length. Psychiatr Genet 17: 195-199, 2007.

14. Bekaert S, De Meyer T, Rietzschel ER, De Buyzere ML, De Bacquer D, Langlois M, Segers P, et al: Telomere length and cardiovascular risk factors in a middle-aged population free of overt cardiovascular disease. Aging Cell 6: 639-647, 2007.

15. von Zglinicki T: Oxidative stress shortens telomeres. Trends Biochem Sci 27: 339-344, 2002.

16. Mirabello L, Huang WY, Wong JY, Chatterjee N, Reding D, Crawford ED, De Vivo I, Hayes RB and Savage SA: The association between leukocyte telomere length and cigarette smoking, dietary and physical variables and risk of prostate cancer. Aging Cell 8: 405-413, 2009.

17. Minamino T, Mitsialis SA and Kourembanas S: Hypoxia extends the life span of vascular smooth muscle cells through telomerase activation. Mol Cell Biol 21: 3336-3342, 2001.

18. Guan JZ, Guan WP, Maeda T and Makino N: Alteration of telomere length and subtelomeric methylation in human endothelial cell under different levels of hypoxia. Arch Med Res 43: 15-20, 2012.
19. Davy P and Allsopp R: Hypoxia Are stem cells in it for the long run? Cell Cycle 10: 206-211, 2011.

20. Feng J, Funk WD, Wang SS, Weinrich SL, Avilion AA, Chiu CP, Adams RR, Chang E, Alisopp RC, Yu J, et al: The RNA component of human telomerase. Science 269: 1236-1241, 1995.

21. Nakamura TM, Morin GB, Chapman KB, Weinrich SL, Andrews WH, Lingner J, Harley CB and Cech TB: Telomerase catalytic subunit homologs from fission yeast and human. Science 277: 955-959, 1997.

22. Harley CB: Telomerases. Pathol Biol (Paris) 42: 342-345, 1994.

23. Cheung AL and Deng W: Telomere dysfunction, genome instability and cancer. Front Biosci 13: 2075-2090, 2008.

24. Fujiki T, Udono M, Kadooka K, Yamashita S, Miura T, Shirahata S and Katakura Y: Regulatory mechanisms of human and mouse telomerase reverse transcriptase gene transcription: Distinct dependency on c-Myc. Cytotechnology 62: 333-339, 2010.

25. Wang Z, Xu J, Geng X and Zhang W: Analysis of DNA methylation status of the promoter of human telomerase reverse transcriptase in gastric carcinogenesis. Arch Med Res 41: 1-6, 2010.

26. Wyatt HD, West SC and Beattie TL: In TERT preting telomerase structure and function. Nucleic Acids Res 38: 5609-5622, 2010.

27. Effros RB: Telomere/telomerase dynamics within the human immune system: Effect of chronic infection and stress. Exp Gerontol 46: 135-140, 2011

28. Jergović M, Tomičević M, Vidović A, Bendelja K, Savić A, Vojvoda V, Rac D, Lovrić-Čavar D, Rabatić S, Jovanovic T and Sabioncello A: Telomere shortening and immune activity in war veterans with posttraumatic stress disorder. Prog Neuropsychopharmacol Biol Psychiatry 54: 275-283, 2014.

29. Effros RB: Replicative senescence in the immune system: Impact of the Hayflick limit on T-cell function in the elderly. Am J Hum Genet 62: 1003-1007, 1998

30. Bertho JM, Demarquay C, Moulian N, Van Der Meeren A, Berrih-Aknin S and Gourmelon P: Phenotypic and immunohistological analyses of the human adult thymus: Evidence for an active thymus during adult life. Cell Immunol 179: 30-40, 1997.

31. Coussens M, Davy P, Brown L, Foster C, Andrews WH, Nagata M and Allsopp R: RNAi screen for telomerase reverse transcriptase transcriptional regulators identifies HIF1alpha as critical for telomerase function in murine embryonic stem cells. Proc Natl Acad Sci USA 107: 13842-13847, 2010.

32. Yang $\mathrm{K}$, Zheng, Deng $\mathrm{X}$, Bai L, Xu Y and Cong YS: Lysophosphatidic acid activates telomerase in ovarian cancer cells through hypoxia-inducible factor-1alpha and the pi3k pathway. J Cell Biochem 105: 1194-1201, 2008. 